\title{
On the Asymptotic Geometric Mean of MIMO Channel Eigenvalues
}

\author{
Jari Salo*, Dominik Seethaler ${ }^{\dagger}$, and Ana Skupch ${ }^{\dagger}$ \\ *Helsinki University of Technology, SMARAD/Radio Laboratory \\ Email: jari.salo@tkk.fi \\ $\dagger$ Vienna University of Technology, Institute of Communications and Radio-Frequency Engineering, \\ Email: \{dominik.seethaler, ana.skupch\}@tuwien.ac.at
}

\begin{abstract}
The geometric mean of the eigenvalues of multipleinput multiple-output (MIMO) channels is a parameter occurring in a variety of instances, including MIMO transceiver design based on the so-called geometric mean decomposition (GMD), and MIMO mutual information at high SNR. In this paper, we derive the asymptotic geometric mean of MIMO channel eigenvalues in the case where the entries of the channel matrix are independent and identically distributed. We demonstrate that the result can be easily extended to channels with an exponential correlation model. As an application of the result, we provide an analytical expression for the asymptotic system capacity of GMD-based transceivers.
\end{abstract}

\section{INTRODUCTION}

The emergence and potential of multiple-input multipleoutput (MIMO) transmission techniques have forced engineers to re-evaluate their traditional approaches to the classical problem of point-to-point communication. In recent years, a number of interesting MIMO techniques have been devised to improve the data throughput and reliability. Yet, despite the great many advances [1], there remain open problems not only in the characterization of the fundamental capacity limits of MIMO channels, but also in the analysis of various MIMO transmission techniques.

Recently, several performance characterization problems revolving around the geometric mean of the MIMO channel eigenvalues have surfaced [2]-[5]. In [2] a transceiver design based on the so-called geometric mean decomposition (GMD) has been proposed. The capacity of the GMD-based design proposed therein depends on the geometric mean of the MIMO channel eigenvalues. A closely related application can be found in [3]. The geometric mean also appears in the analysis of the vector perturbation technique introduced in [4]. In [5], it is shown that the ratio of geometric and arithmetic means is a natural measure for characterizing the capacity degradation due to dispersion of the eigenvalues. It is also well-known that the logarithm of the geometric mean of the MIMO channel eigenvalues provides a simple approximation to the high-SNR channel capacity with uniform transmit power allocation [6].

Let $\mathbf{H}$ denote a $K \times L$ matrix whose zero-mean entries are independent and identically distributed (iid) random variables with unit variance. The asymptotic $(K \rightarrow \infty)$ geometric mean of the positive eigenvalues of $\frac{1}{L} \mathbf{H} \mathbf{H}^{H}$ has been derived in [7] for arbitrary ratio parameter $\beta=\frac{L}{K}$ by direct integration over the Marčenko-Pastur distribution [8]. The case of square $\mathbf{H}(\beta=1)$ was derived for Gaussian $\mathbf{H}$ in [9] using a limit argument. The result therein however coincides with the asymptotic geometric mean of a larger class of random matrices whose empirical eigenvalue distribution converges to the Marčenko-Pastur law. For certain distributions the exact expected value of logarithm of the geometric mean is known for finite matrix sizes. For example, if $\mathbf{H}$ is matrix variate Gaussian, i.e., $\frac{1}{L} \mathbf{H} \mathbf{H}^{H}$ is Wishart, the expected logdeterminant of $\frac{1}{L} \mathbf{H} \mathbf{H}^{H}$ is well-known to be given by a sum of digamma functions [6]. This result, while exact, provides little insight on how the geometric mean behaves as the ratio $L / K$ varies.

In this paper we derive the asymptotic geometric mean of the eigenvalues of the matrix quadratic form $\frac{1}{L} \mathbf{H} \mathbf{H}^{H}$ by a method different from [7]. The proof is based on a simple limiting argument and avoids integration over the MarčenkoPastur law. The case of exponentially correlated matrix entries is also briefly discussed. As an application, we analyze the system capacity of the recently proposed GMD-based transceivers [2].

The paper is organized as follows. Section II gives the main result with two proofs. The application of the main result to the capacity of GMD-based transceivers can be found in Section III. Numerical experiments in Section IV confirm the validity of our analytical results. Section V concludes the paper.

Notation: A matrix, vector, and scalar are denoted with $\mathbf{A}$, $\mathbf{a}$, and $a$, respectively. Conjugate transpose of $\mathbf{A}$ is denoted with $\mathbf{A}^{H}$, and the $n \times n$ identity matrix with $\mathbf{I}_{n}$. Determinant of a square matrix $\mathbf{A}$ is denoted with $|\mathbf{A}| . \mathrm{E}[X]$ stands for the expected value of the random variable $X$. The 'Big Oh' notation $f(n)=O(g(n))$ means that there exists some constant $c$ such that, for large enough $n, c g(n)$ is an upper bound on $f(n)$.

\section{ASYMPTOTIC GEOMETRIC MEAN}

Consider a real or complex $K \times L$ matrix $\mathbf{H}$, whose entries are iid random variables with zero-mean and unit variance. We are interested in the asymptotic limit, i.e. $K \rightarrow \infty$ while

$$
\beta \triangleq \frac{L}{K} \geq 1
$$


is held constant, of the geometric mean of the eigenvalues $\left\{\lambda_{k}\right\}_{k=1}^{K}$ of the matrix

$$
\mathbf{G} \triangleq \frac{1}{L} \mathbf{H H}^{H}
$$

given by

$$
m_{g} \triangleq \lim _{K \rightarrow \infty}\left(\prod_{k=1}^{K} \lambda_{k}\right)^{\frac{1}{K}}=\lim _{K \rightarrow \infty}|\mathbf{G}|^{\frac{1}{K}} .
$$

We stress that $m_{g}$ denotes the asymptotic geometric mean. As a technical side condition, we also assume that the fourth moments of the entries of $\mathbf{H}$ decay as $O\left(L^{-2}\right)$.

\section{A. Main result}

Result 1: With the assumptions made above, the asymptotic geometric mean of the eigenvalues of the matrix $\mathbf{G}=\frac{1}{L} \mathbf{H} \mathbf{H}^{H}$ is given by

$$
m_{g}=\left(\frac{\beta}{\beta-1}\right)^{\beta-1} \frac{1}{e}, \quad \beta \geq 1 .
$$

It follows that $m_{g}$ varies between $m_{g}=1 / e$ (for $\beta \rightarrow 1$ ) and $m_{g}=1$ (for $\beta \rightarrow \infty$ ) since

$$
\lim _{\beta \rightarrow 1}\left(\frac{\beta}{\beta-1}\right)^{\beta-1}=1, \quad \lim _{\beta \rightarrow \infty}\left(\frac{\beta}{\beta-1}\right)^{\beta-1}=e .
$$

This result generalizes the case $\beta=1$ in [9], where it was shown that for square $\mathbf{H}$ one obtains

$$
m_{g}=\frac{1}{e} .
$$

Note that (1) is the asymptotic geometric mean of the eigenvalues of $\frac{1}{K} \mathbf{H}^{H} \mathbf{H}$ for $K \geq L$ if $\beta$ is replaced with $\beta^{\prime}=1 / \beta=K / L$.

\section{B. Direct proof}

It is known that, as $K \rightarrow \infty$, the empirical distribution of the eigenvalues of $\mathbf{G}$ converges to the Marčenko-Pastur law [8]

$$
f(\lambda)=\frac{\sqrt{(\lambda-a)(b-\lambda)}}{2 \pi \beta \lambda}, \quad a \leq \lambda \leq b,
$$

where

$$
a=(\sqrt{\beta}-1)^{2}, \quad b=(\sqrt{\beta}+1)^{2} .
$$

In the sequel, it will be more convenient to work with the logarithm of $m_{g}$, i.e. $\ln m_{g}$, since this allows us to convert a finite summation to a definite integral at the large- $K$ limit, and then exploit the Marčenko-Pastur law.

Towards this end, we write

$$
\begin{aligned}
\ln m_{g} & =\lim _{K \rightarrow \infty} \frac{1}{K} \ln (|\mathbf{G}|) \\
& =\lim _{K \rightarrow \infty} \frac{1}{K} \sum_{k=1}^{K} \ln \left(\lambda_{k}\right) \\
& =\int_{a}^{b} \ln (\lambda) f(\lambda) \mathrm{d} \lambda \\
& =\int_{a}^{b} \frac{\sqrt{(\lambda-a)(b-\lambda)}}{2 \pi \beta \lambda} \ln \lambda \mathrm{d} \lambda,
\end{aligned}
$$

where (6) follows from (5) because, by the assumptions on the statistics of $\mathbf{H}$, at the large- $K$ limit the average converges to the Marčenko-Pastur law given in (3). Thus, the integral we need to evaluate is

$$
\int_{a}^{b} \frac{\sqrt{(\lambda-a)(b-\lambda)}}{\lambda} \ln \lambda \mathrm{d} \lambda,
$$

where $a$ and $b$ were defined in (4). After substitution $\lambda=$ $1+\beta-2 \sqrt{\beta} \cos x, x \in[0, \pi],(8)$ assumes the form

$$
4 \beta \int_{0}^{\pi} \frac{\sin ^{2} x \ln (1+\beta-2 \sqrt{\beta} \cos x)}{1+\beta-2 \sqrt{\beta} \cos x} \mathrm{~d} x .
$$

From $2 \sin ^{2} x=1-\frac{1}{2}\left(e^{j 2 x}+e^{-j 2 x}\right)$, we have

$$
2 \beta \int_{0}^{\pi} \frac{\left[1-\frac{1}{2}\left(e^{j 2 x}+e^{-j 2 x}\right)\right] \ln (1+\beta-2 \sqrt{\beta} \cos x)}{1+\beta-2 \sqrt{\beta} \cos x} \mathrm{~d} x,
$$

which has been evaluated in the Appendix of [10] and in [7] using a shorter Poisson integral approach. Using the results therein, the logarithm of the asymptotic geometric mean is then

$$
\ln m_{g}=(\beta-1) \ln \left(\frac{\beta}{\beta-1}\right)-1,
$$

which gives (1).

\section{Indirect (probabilistic) proof}

The direct proof given above hinges on the non-obvious substitution $x$, and on the subsequent integration of (9), given in [7] and [10]. In what follows, we present an alternative proof which is based on a totally different approach. The idea is to examine the limit of the logarithm of the geometric mean of eigenvalues of $\mathbf{G}=\frac{1}{L} \mathbf{H} \mathbf{H}^{H}$ in the case where the entries of $\mathbf{H}$ are iid complex Gaussian random variables with zero-mean and unit variance, i.e, the distribution of $\mathbf{G}$ is complex Wishart. As $K \rightarrow \infty$, the empirical distribution of the eigenvalues of G converges almost surely to the Marčenko-Pastur law, and hence from standard convergence theorems [11] it follows that the expected value of $\frac{1}{K} \ln |\mathbf{G}|$ will coincide with (7) in the large- $K$ limit. For finite $K$ we can write [6], [8]

$$
\mathrm{E}\left[\ln |\mathbf{G}|^{\frac{1}{K}}\right]=-\ln L+\frac{1}{K} \sum_{k=0}^{K-1} \psi(L-k),
$$

where, for positive integer $n$,

$$
\psi(n)=-\gamma+H_{n-1},
$$

is the digamma function, $\gamma \approx 0.5772$ is the Euler-Mascheroni constant, and

$$
H_{k}=\sum_{m=1}^{k} \frac{1}{m}, \quad H_{0}=0,
$$

is the $k$ th harmonic number. The main obstacle is to evaluate the sum of digamma functions in (10). We can assume without loss in generality that $L \geq K$. From (11) and (12) we can write

$$
\sum_{k=0}^{K-1} \psi(L-k)=-K \gamma+\sum_{n=1}^{K} H_{L-n}
$$


We also have

$$
\begin{aligned}
\sum_{n=1}^{L-1} H_{n} & =\sum_{n=1}^{K} H_{L-n}+\sum_{n=1}^{L-K-1} H_{n} \\
& =L H_{L}-L,
\end{aligned}
$$

where we used the easily proven sum [12]

$$
\sum_{k=1}^{n-1} H_{k}=n H_{n}-n
$$

From (13)-(15) it is clear that

$$
\sum_{n=1}^{K} H_{L-n}=L H_{L}-(L-K) H_{L-K}-K .
$$

Therefore, with $L=\beta K$,

$$
\frac{1}{K} \sum_{k=0}^{K-1} \psi(L-k)=\beta H_{\beta K}-(\beta-1) H_{(\beta-1) K}-1-\gamma .
$$

For large $n$,

$$
H_{n}=\gamma+\ln n+O\left(n^{-1}\right) .
$$

Using (17), for large $K$, (16) becomes

$$
\begin{aligned}
& \frac{1}{K} \sum_{k=0}^{K-1} \psi(L-k) \\
= & \beta \ln (\beta K)-(\beta-1) \ln [(\beta-1) K]-1+O\left(K^{-1}\right) .
\end{aligned}
$$

Combining (18) and (10) with $-\ln L=-\ln (\beta K)$, we obtain the final result as $K \rightarrow \infty$

$$
\ln m_{g}=(\beta-1) \ln \left(\frac{\beta}{\beta-1}\right)-1,
$$

which again gives (1).

We have therefore proved Result 1 indirectly as the limiting value of the Wishart distributed $\mathbf{G}$. The proof bypasses the cumbersome definite integral over the Marčenko-Pastur distribution. The idea of the proof may be applicable to $\mathbf{H}$ with dependent entries to obtain asymptotic results that would be difficult to derive directly.

\section{Impact of Correlation}

In general, one-sided correlation adds to $\ln m_{g}$ a penalty term that depends on the asymptotic correlation structure of the channel. As an example, if $\mathbf{H}_{c}=\sqrt{\mathbf{R}} \mathbf{H}$, where the $(i, j)$ th entry of $\mathbf{R}$ is $\mu^{|i-j|}, \mu \in(0,1)$, it can be shown that the asymptotic geometric mean of the eigenvalues of $\mathbf{G}_{c} \triangleq \frac{1}{L} \mathbf{H}_{c} \mathbf{H}_{c}^{H}$ is

$$
\begin{aligned}
m_{g, c} & =\left(\frac{\beta}{\beta-1}\right)^{\beta-1} \frac{1-\mu^{2}}{e} \\
& =\left(1-\mu^{2}\right) m_{g} .
\end{aligned}
$$

Here, $\mathbf{H}_{c}=\sqrt{\mathbf{R}} \mathbf{H}$ could correspond to a MIMO channel modeled by the popular exponential correlation model at the receiver side. The effect of this correlation is captured by (19) in a very simple way. To prove (19), one can first write $\ln \left|\mathbf{G}_{c}\right|^{\frac{1}{K}}=\ln |\mathbf{R}|^{\frac{1}{K}}+\ln |\mathbf{G}|^{\frac{1}{K}}$, and then evaluate the large- $K$ limit of the first term based on the asymptotic eigenvalue distribution of Toeplitz matrices [14]. If the correlation is modelled in a more general way (in the sense that the geometric means of the correlation and the iid terms cannot be separated), the idea of the probabilistic proof could still be helpful.

\section{CAPACITY OF GMD-BASED MIMO TRANSMISSION}

\section{A. MIMO System Model}

We consider a MIMO communications systems, where $K$ and $L$ now refer to the number of receive and transmit antennas, respectively. For a frequency-flat communication channel, the channel input $\mathbf{x} \in \mathbb{C}^{L \times 1}$ and channel output $\mathbf{y} \in \mathbb{C}^{K \times 1}$ are related as

$$
\mathbf{y}=\mathbf{H x}+\mathbf{n},
$$

where $\mathbf{n} \in \mathbb{C}^{K \times 1}$ denotes additive noise. We assume that $\mathbf{n}$ and $\mathbf{x}$ have multivariate circularly symmetric complex Gaussian distribution with per-dimension variances of $\sigma_{n}^{2}$ and $\sigma_{x}^{2}$, respectively. Furthermore, we assume that the entries of the channel matrix $\mathbf{H}$ are iid Gaussian with zero-mean and unit variance. In this pratically important case, $\mathbf{G}=\frac{1}{L} \mathbf{H} \mathbf{H}^{H}$ satisfies the assumptions of Result 1 and the assumptions of the Marčenko-Pastur law. The average signal-to-noise ratio (SNR) per receive antenna is defined as

$$
\mathrm{SNR}=\frac{\mathrm{E}\left[\mathbf{x}^{H} \mathbf{x}\right]}{\sigma_{n}^{2}} .
$$

\section{B. GMD-Based Transmission}

In conventional MIMO systems employing V-BLAST (i.e. decision-feedback) detection (e.g. [15]) or TomlinsonHarashima (TH) precoding (e.g. [16]), the overall error performance is usually dominated by the equivalent subchannel that has the lowest SNR. This worst subchannel SNR can be maximized using GMD-based transceiver design [2], which can be seen as a generalized QR-type decomposition of the channel matrix. In combination with V-BLAST detection or TH precoding, the MIMO channel is transformed into equal power parallel subchannels; the gain of each subchannel is given by the geometric mean of the eigenvalues of the matrix $\mathbf{H H}^{H}$. This reduces the degrading effect of low-SNR subchannels, and improves the overall error performance.

\section{Asymptotic System Capacity of GMD-Based Transmission}

With (1) we are now able to provide an analytic expression for the asymptotic system capacity of GMD-based transmission.

The system capacity for GMD-based transceiver design is given by [2]

$$
C_{\mathrm{GMD}}=K \log _{2}\left(1+\frac{\mathrm{SNR}}{L} m_{g, K}\right),
$$


where $m_{g, K}$ refers to the geometric mean of the eigenvalues of the $K \times K$ matrix $\mathbf{H H}^{H}$, i.e.

$$
m_{g, K} \triangleq\left|\mathbf{H H}^{H}\right|^{\frac{1}{K}} .
$$

Here, we assume that $K \leq L$ and that the $K \times L$ channel matrix $\mathbf{H}$ has full rank (almost surely). The channel $\mathbf{H}$ is random, and so are $m_{g, K}$ and $C_{\mathrm{GMD}}$. However, as $K \rightarrow \infty$ while $\beta=L / K$ is held constant, $m_{g, K} / L$ (i.e. the geometric mean of the eigenvalues of $\mathbf{G}=\frac{1}{L} \mathbf{H} \mathbf{H}^{H}$ ) tends almost surely to $m_{g}$ defined in (1), and hence the system capacity per subchannel $C_{\mathrm{GMD}} / K$ tends almost surely to

$$
\begin{aligned}
\frac{C_{\mathrm{GMD}}^{\infty}}{K} & =\log _{2}\left(1+\operatorname{SNR} m_{g}\right) \\
& =\log _{2}\left(1+\frac{\mathrm{SNR}}{e}\left(\frac{\beta}{\beta-1}\right)^{\beta-1}\right) .
\end{aligned}
$$

Here $m_{g}$ can be seen simply as a $\beta$-dependent SNR gain that varies between $1 / e$ (for $\beta \rightarrow 1$ ) and 1 (for $\beta \rightarrow \infty$ ). Evidently, if we replace $m_{g}$ with $m_{g, c}=\left(1-\mu^{2}\right) m_{g}$ [see (19)], we immediately obtain the asymptotic system capacity for GMD-based transeiver design with exponential correlation at the receiver side. Via the factor $1-\mu^{2}$, the correlation parameter $\mu$ directly determines the associated capacity loss compared to the iid case.

It is readily shown that in the case when there are more receive than transmit antennas (i.e. $K>L$ ), (20) holds for $C_{\mathrm{GMD}}^{\infty} / L$ if we replace $\beta$ with $\beta^{\prime}=1 / \beta=K / L$ and SNR with $\beta^{\prime}$ SNR.

\section{NUMERICAL EXAMPLES AND DISCUSSION}

\section{A. Asymptotic Geometric Mean}

To test the validity of Result 1 we generated a large number of $K \times L$ matrices whose entries were iid zero-mean Gaussian with unit variance. We then computed the geometric mean of the eigenvalues of the $K \times K$ matrix $\mathbf{G}$ for all realizations, and averaged the result. Sample standard deviation from the average was also computed. The procedure was repeated for several values of $L$. The result for $K=8$ is shown in Fig. 1 . It can be seen that the asymptotic result provides an excellent approximation to the average geometric mean results even for the moderately small $K=8$. Moreover, the asymptotic geometric mean is also a good approximation for any given realization, as evidenced by the fairly small standard deviation. This is an expected result since the empirical distribution of the eigenvalues of $\mathbf{G}$ converges to the limiting case rather quickly as $K$ increases [8].

\section{B. Capacity of GMD-Based Transmission}

Fig. 2 compares the analytical result (20) to capacity simulated using finite dimensional channel matrices. Samples of $\mathbf{H}$ were generated according to the iid Gaussian MIMO channel model described in Subsection III-A with $K=8$. The standard deviation from the average capacity is also shown. It can be seen that the asymptotic result serves as an accurate, yet simple approximation even for moderate number of antennas.

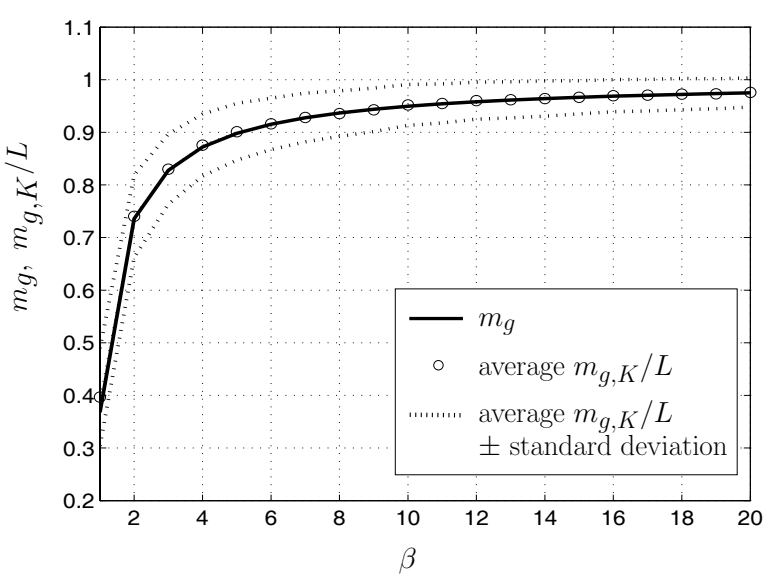

Fig. 1. Numerical evaluation of the asymptotic geometric mean versus $\beta$ for $K=8$; average (finite $K$ ) geometric mean $L^{-1} m_{g, K}$ with \pm standard deviations as well as the corresponding asymptotic result are shown.

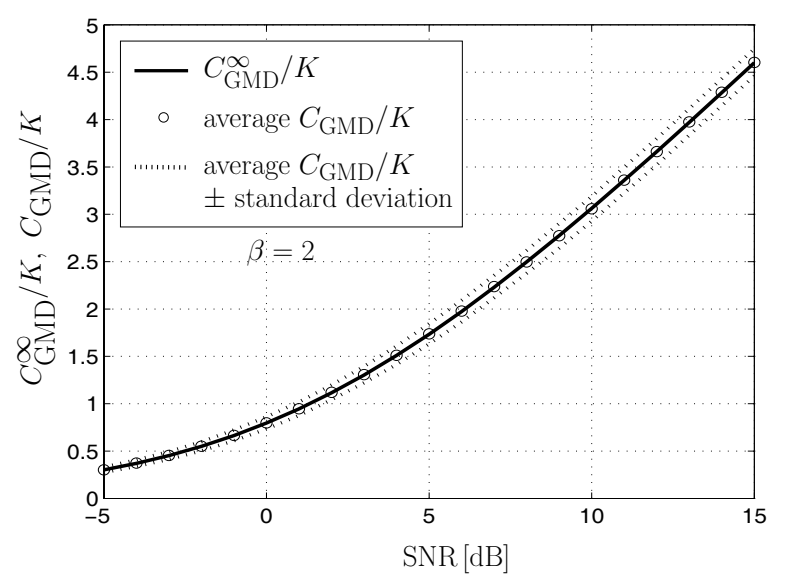

Fig. 2. Numerical evaluation of the GMD capacity versus SNR for $K=8$, $\beta=2$; average capacity with \pm standard deviations and the corresponding asymptotic result are shown.

We now compare the GMD capacity with the channel capacity for uniform power loading (when no channel state information is available at the transmitter), defined as [1]

$$
C=\mathrm{E}\left[\log _{2}\left|\mathbf{I}_{K}+\frac{\mathrm{SNR}}{L} \mathbf{H H}^{H}\right|\right],
$$

where the expectation is with respect to the channel distribution. The analytic result for the corresponding asymptotic capacity $C^{\infty} / K$ can be found in [10], [13]. Note that the result for $C^{\infty} / K$ therein has a much more complicated form than the GMD capacity in (20). Figure 3 (a) and (b) show the asymptotic capacity results $C^{\infty} / K$ and $C_{\mathrm{GMD}}^{\infty} / K$ versus SNR (for $\beta=2$ ) and versus $\beta$ (for $\mathrm{SNR}=5 \mathrm{~dB}$ ), respectively. It is seen that GMD-based transmission is inferior especially at low SNR and small $\beta$. On the other hand, for large $\beta$ or high SNR we obtain the following. 
- High SNR, fixed $\beta$ : Evidently, for high SNR we can approximate

$$
\frac{C_{\mathrm{GMD}}^{\infty}}{K} \approx \log _{2}\left(\mathrm{SNR} m_{g}\right)
$$

where the approximation error vanishes as SNR $\rightarrow \infty$. It is easy to show that, for large number of antennas, this coincides with the high-SNR approximation of (21). It follows that GMD-based transceivers are capacity optimal for high SNR (cf. Figure 3 (a)). This has been discussed also in [2].

- Large $\beta$, fixed SNR: For $\beta \rightarrow \infty$ we have from (2) $m_{g} \rightarrow$ 1 , and thus

$$
\lim _{\beta \rightarrow \infty} \frac{C_{\mathrm{GMD}}^{\infty}}{K}=\log _{2}(1+\mathrm{SNR}) .
$$

This is the capacity of a single-input single-output AWGN channel, and also, as is well-known [10], the large- $\beta$ limit of the asymptotic per-dimension channel capacity associated with (21). Thus, we can conclude that, as expected, GMD-based transceivers are asymptotically capacity optimal for large $\beta$ (cf. Figure 3 (b)).

\section{CONCLUSiON}

Using a simple limiting approach, we derived the large- $K$ limit of the geometric mean of the eigenvalues of $\frac{1}{L} \mathbf{H H}$ in the case where the $K \times L$ matrix $\mathbf{H}$ has zero-mean iid elements with unit variance and $\frac{L}{K}$ is fixed. We pointed out how the result can be directly extended to an exponetially correlated case. As an application, we obtained a simple expression for the asymptotic capacity of transceivers based on the geometric mean decomposition.

\section{ACKNOWLEDGMENT}

This work was supported by WWTF grant MA 44 (MOHAWI) and Academy of Finland. The authors would like to thank Jan Eriksson for his helpful comments, and Walid Hachem for pointing out [7].

\section{REFERENCES}

[1] E. Telatar, "Capacity of multi-antenna Gaussian channels," European Transactions on Telecommunications, vol. 10, no. 6, pp. 585-596, November 1999.

[2] Y. Jiang, J. Li, and W. W. Hager, "Joint transceiver design for MIMO communications using geometric mean decomposition," IEEE Trans. Signal Processing, vol. 53, no. 10, pp. 3791-3803, Oct. 2005.

[3] J.-K. Zhang, A. Kavcic, and K. M. Wong, "Equal-diagonal QR decomposition and its application to precoder design for successive-cancellation detection," IEEE Trans. Inform. Theory, vol. 51, no. 1, pp. 154-172, Jan. 2005.

[4] B. M. Hochwald, C. B. Peel, and A. L. Swindlehurst, "A vectorperturbation technique for near-capacity multiantenna multiuser communication - part II: Perturbation," IEEE Trans. Commun., vol. 53, no. 3 , pp. 537-544, Mar. 2005.

[5] J. Salo, P. Suvikunnas, H. M. El-Sallabi, and P. Vainikainen, "Some results on MIMO capacity: the high SNR case," in Proc. Global Telecomm. Conf., Dallas, USA, Dec. 2004.

[6] A. Grant, "Rayleigh fading multiple-antenna channels," EURASIP Journal on Applied Signal Processing, vol. 2002, no. 3, pp. 316-329, Mar. 2002.

[7] Z. D. Bai and J. W. Silverstein, "CLT for linear spectral statistics of large-dimensional sample covariance matrices," The Annals of Probability, vol. 32, no. 1A, pp. 553-605, 2004. (a)

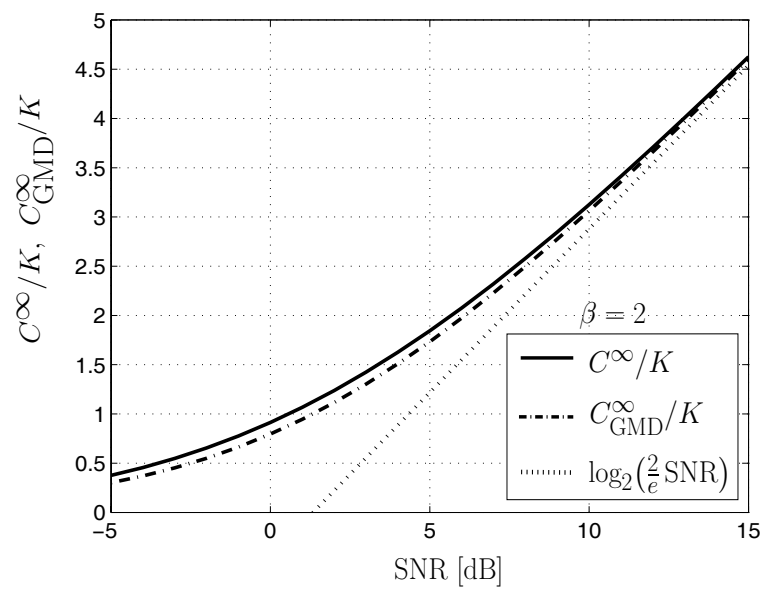

(b)

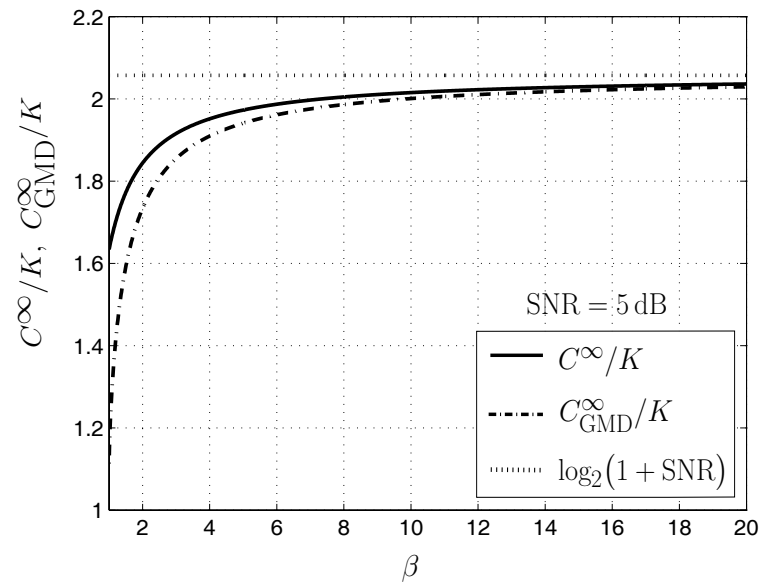

Fig. 3. Asymptotic channel capacity with equal power loading [10], [13] and asymptotic GMD capacity (20) per subchannel: (a) versus SNR with $\beta=2$, and (b) versus $\beta$ with SNR $=5 \mathrm{~dB}$.

[8] A. M. Tulino and S. Verdú, Random Matrix Theory and Wireless Communications, ser. Foundations and Trends in Communications and Information Theory. now Publishers Inc., 2004, vol. 1, no. 1.

[9] G. M. Cicuta and M. L. Mehta, "Probability density of determinants of random matrices," J. Phys. A: Math. Gen., vol. 33, pp. 8029-8035, 2000.

[10] P. B. Rapajic and D. Popescu, "Information capacity of a random signature multiple-input multiple-output channel," IEEE Trans. Commun., vol. 48, no. 8, pp. 1245-1248, Aug. 2000.

[11] R. J. Serfling, Approximation Theorems of Mathematical Statistics. John Wiley \& Sons, Inc., 1980.

[12] R. L. Graham, D. E. Knuth, and O. Patashnik, Concrete Mathematics. Addison-Wesley, 1990.

[13] C.-N. Chuah, D. N. Tse, J. M. Kahn, and R. A. Valanzuela, "Capacity scaling in MIMO wireless systems under correlated fading," IEEE Trans. Inform. Theory, vol. 48, no. 3, pp. 637-650, Mar. 2002.

[14] R. M. Gray, Toeplitz and Circulant Matrices: A review, ser. Foundations and Trends in Communications and Information Theory. now Publishers, Inc., 2006, vol. 2, no. 3.

[15] G. D. Golden, C. J. Foschini, R. A. Valenzuela, and P. W. Wolniansky, "Detection algorithm and initial laboratory results using V-BLAST space-time communications architecture," Elect. Lett., vol. 35, pp. 14 16, Jan. 1999.

[16] C. Windpassinger, R. F. H. Fischer, T. Vencel, and J. B. Huber, "Precoding in multi-antenna and multi-user communications," IEEE Trans. Wireless Comm., vol. 3, no. 4, pp. 1305-1316, July 2004. 